\title{
Foliations on modular curves
}

\author{
Igor V. Nikolaev* \\ In memory of Marco Brunella
}

\begin{abstract}
It is proved, that a foliation on a modular curve given by the vertical trajectories of holomorphic differential corresponding to the Hecke eigenform is either the Strebel foliation or the pseudo-Anosov foliation.
\end{abstract}

Key words and phrases: Hecke eigenforms, singular foliations

MSC: 11F12 (automorphic forms); $57 R 30$ (foliations)

\section{Introduction}

Let $N>1$ be a natural number and consider a finite index subgroup of the modular group given by the formula:

$$
\Gamma_{0}(N)=\left\{\left(\begin{array}{ll}
a & b \\
c & d
\end{array}\right) \in S L(2, \mathbb{Z}) \mid c \equiv 0 \bmod N\right\} .
$$

Let $\mathbb{H}=\{z=x+i y \in \mathbb{C} \mid y>0\}$ be the upper half-plane and let $\Gamma_{0}(N)$ act on $\mathbb{H}$ by linear fractional transformations; consider the orbifold $\mathbb{H} / \Gamma_{0}(N)$. To compactify the orbifold at the cusps, one adds a boundary to $\mathbb{H}$, so that $\mathbb{H}^{*}=\mathbb{H} \cup \mathbb{Q} \cup\{\infty\}$ and the compact Riemann surface $X_{0}(N)=\mathbb{H}^{*} / \Gamma_{0}(N)$ is called a modular curve. The holomorphic functions $f(z)$ on $\mathbb{H}$ that vanish at the cusps and such that

$$
f\left(\frac{a z+b}{c z+d}\right)=(c z+d)^{2} f(z), \quad \forall\left(\begin{array}{ll}
a & b \\
c & d
\end{array}\right) \in \Gamma_{0}(N),
$$

\footnotetext{
*Partially supported by NSERC.
} 
are called cusp forms of weight two; the (complex linear) space of such forms will be denoted by $S_{2}\left(\Gamma_{0}(N)\right)$. The formula $f(z) \mapsto \omega=f(z) d z$ defines an isomorphism $S_{2}\left(\Gamma_{0}(N)\right) \cong \Omega_{h o l}\left(X_{0}(N)\right)$, where $\Omega_{h o l}\left(X_{0}(N)\right)$ is the space of holomorphic differentials on the Riemann surface $X_{0}(N)$. Note that $\operatorname{dim}_{\mathbb{C}}\left(S_{2}\left(\Gamma_{0}(N)\right)=\operatorname{dim}_{\mathbb{C}}\left(\Omega_{\text {hol }}\left(X_{0}(N)\right)=g\right.\right.$, where $g=g(N)$ is the genus of the surface $X_{0}(N)$. A Hecke operator, $T_{n}$, acts on $S_{2}\left(\Gamma_{0}(N)\right)$ by the formula $T_{n} f=\sum_{m \in \mathbb{Z}} \gamma(m) q^{m}$, where $\gamma(m)=\sum_{a \mid G C D(m, n)} a c_{m n / a^{2}}$ and $f(z)=\sum_{m \in \mathbb{Z}} c(m) q^{m}$ is the Fourier series of the cusp form $f$ at $q=e^{2 \pi i z}$. For $(n, N)=1$ it is known that $T_{n}$ is a self-adjoint linear operator on the vector space $S_{2}\left(\Gamma_{0}(N)\right)$ endowed with the Petersson inner product; the algebra $\mathbb{T}_{N}:=\mathbb{Z}\left[T_{1}, T_{2}, \ldots\right]$ is a commutative algebra. Any cusp form $f_{N} \in S_{2}\left(\Gamma_{0}(N)\right)$ that is an eigenvector for one of $T_{n}$ is referred to as a Hecke eigenform; such an eigenform is called rational whenever its Fourier coefficients $c(m) \in \mathbb{Z}$ after the normalization $c(1)=1$.

Let $\phi=\Re(\omega)$ be the real part of $\omega$; it is a closed form on the surface $X_{0}(N)$. (Alternatively, one can take for $\phi$ the imaginary part of $\omega$.) Note that $\phi$ is well-defined modulo a real multiple $C$; indeed, because $f_{N}(z)=$ $\sum_{m \in \mathbb{Z}} c(m) q^{m}$ and $c(m) \in \mathbb{R}$ it is easy to deduce that for each eigenform $C f_{N}(z)$ the constant $C \in \mathbb{R}$. By $\mathcal{F}$ we understand a measured foliation given by trajectories of the closed form $\phi$; we address the following

Classification problem. Find the topological types of foliation $\mathcal{F}_{N}$ given by the Hecke eigenform $f_{N}$.

Recall that measured foliation $\mathcal{F}$ is called a Strebel foliation, if all but a finite number of its leaves are closed [Strebel 1984] [6]; the Strebel foliation is invariant of a parabolic automorphism of the surface. The measured foliation $\mathcal{F}$ is called a pseudo-Anosov foliation, if it is minimal and invariant of a hyperbolic (pseudo-Anosov) automorphism of the surface [Casson \& Bleiler 1988] [2]; our main result can be expressed as follows.

Theorem 1 The foliation $\mathcal{F}_{N}$ is topologically conjugate to a pseudo-Anosov foliation unless $f_{N}$ is a rational eigenform, in which case it is topologically conjugate to a Strebel foliation.

The article is organized as follows. All preliminary results are introduced in Section 2; all proofs are given in Section 3. 


\section{Preliminaries}

\section{$2.1 \quad$ Measured foliations}

By a $p$-dimensional, class $C^{r}$ foliation of an $m$-dimensional manifold $M$ one understands a decomposition of $M$ into a union of disjoint connected subsets $\left\{\mathcal{L}_{\alpha}\right\}_{\alpha \in A}$, called the leaves of the foliation. The leaves must satisfy the following property: Every point in $M$ has a neighborhood $U$ and a system of local class $C^{r}$ coordinates $x=\left(x^{1}, \ldots, x^{m}\right): U \rightarrow \mathbb{R}^{m}$ such that for each leaf $\mathcal{L}_{\alpha}$, the components of $U \cap \mathcal{L}_{\alpha}$ are described by the equations $x^{p+1}=$ Const $, \ldots, x^{m}=$ Const. Such a foliation is denoted by $\mathcal{F}=\left\{\mathcal{L}_{\alpha}\right\}_{\alpha \in A}$. The number $q=m-p$ is called a codimension of the foliation $\mathcal{F}$, see [Lawson 1974] [5] p.370. The codimension $q C^{r}$-foliations $\mathcal{F}_{0}$ and $\mathcal{F}_{1}$ are said to be $C^{s}$-conjugate $(0 \leq s \leq r)$ if there exists a diffeomorphism of $M$, of class $C^{s}$, which maps the leaves of $\mathcal{F}_{0}$ onto the leaves of $\mathcal{F}_{1}$. If $s=0, \mathcal{F}_{0}$ and $\mathcal{F}_{1}$ are topologically conjugate [Lawson 1974] [5], p.388; we shall write such foliations as $\mathcal{F}_{0} \cong \mathcal{F}_{1}$. The foliation $\mathcal{F}$ is called singular if the codimension $q$ of the foliation depends on the leaf. We further assume that $q$ is constant for all but a finite number of leaves. Such a set of the exceptional leaves will be denoted by $\operatorname{Sing} \mathcal{F}:=\left\{\mathcal{L}_{\alpha}\right\}_{\alpha \in F}$, where $|F|<\infty$. Note that in the case $F$ is an empty set, one gets the usual definition of a (non-singular) foliation. A quick example of the singular foliations is given by the trajectories of a non-trivial differential form on the manifold $M$, which vanish in a finite number of points of $M$; the set of zeroes of such a form corresponds to the exceptional leaves of the foliation. A measured foliation $\mathcal{F}$ on the surface $X$ is the partition of $X$ into the singular points $x_{1}, \ldots, x_{n}$ of order $k_{1}, \ldots, k_{n}$ and regular leaves (1dimensional submanifolds). On each open cover $U_{i}$ of $X-\left\{x_{1}, \ldots, x_{n}\right\}$ there exists a non-vanishing real-valued closed 1-form $\phi_{i}$ such that: (i) $\phi_{i}= \pm \phi_{j}$ on $U_{i} \cap U_{j}$; (ii) at each $x_{i}$ there exists a local chart $(u, v): V \rightarrow \mathbb{R}^{2}$ such that for $z=u+i v$, we have $\phi_{i}=\operatorname{Im}\left(z^{\frac{k_{i}}{2}} d z\right)$ on $V \cap U_{i}$ for some branch of $z^{\frac{k_{i}}{2}}$. The pair $\left(U_{i}, \phi_{i}\right)$ is called an atlas for the measured foliation $\mathcal{F}$. Finally, a measure $\mu$ is assigned to each segment $\left(t_{0}, t\right) \in U_{i}$, which is transverse to the leaves of $\mathcal{F}$, via the integral $\mu\left(t_{0}, t\right)=\int_{t_{0}}^{t} \phi_{i}$. The measure is invariant along the leaves of $\mathcal{F}$, hence the name. Note that the measured foliation can have singular points with an odd number of the saddle sections (half-integer index); those cannot be continuously oriented along the leaves and therefore cannot be given by the trajectories of a closed form $\phi$. However, each measured foliation can be rendered oriented by taking if necessary the double cover of $X$ 
ramified at the singular points of the half-integer index; further we work with the foliations given by trajectories of a closed form $\phi$. Let $\left\{\gamma_{1}, \ldots, \gamma_{n}\right\}$ be a basis in the relative homology group $H_{1}(X, \operatorname{Sing} \mathcal{F} ; \mathbb{Z})$, where $\operatorname{Sing} \mathcal{F}$ is the set of singular points of the foliation $\mathcal{F}$; here $n=2 g+m-1$, where $g$ is the genus of $X$ and $m=|\operatorname{Sing}(\mathcal{F})|$. (In what follows it will be shown that $m=1$ and an involution on $H_{1}(X, \operatorname{Sing} \mathcal{F} ; \mathbb{Z})$ will imply that $n=g$, see proof of lemma 5.) Let $\lambda_{i}$ be the periods of $\phi$ against the basis $\gamma_{i}$. By a Jacobian of measured foliation $\mathcal{F}$ we understand a $\mathbb{Z}$-module $\operatorname{Jac}(\mathcal{F}):=\mathbb{Z} \lambda_{1}+\ldots+\mathbb{Z} \lambda_{n}$ regarded as a subset of the real line $\mathbb{R}$. The following lemma describes basic properties of the Jacobians; it will be proved in Section 3.1.

Lemma 1 For each uniquely ergodic measured foliation $\mathcal{F}$ the following is true:

(i) Jac $(\mathcal{F})$ is a well-defined $\mathbb{Z}$-module of the real line $\mathbb{R}$;

(ii) if $\mathcal{F} \cong \mathcal{F}^{\prime}$ are topologically conjugate foliations, then Jac $\left(\mathcal{F}^{\prime}\right)=$ $\mu \operatorname{Jac}(\mathcal{F})$ for a constant $\mu>0$, i.e. the projective class of Jac $(\mathcal{F})$ is an invariant of topological conjugation.

\subsection{Automorphisms of surfaces}

Let $\varphi: X \rightarrow X$ be an orientation-preserving automorphism of a compact surface $X$; the composition of (the isotopy classes of) such automorphisms define a countable group denoted by $\operatorname{Mod}(X)$. The measured foliations play an outstanding rôle in the classification of surface automorphisms; to give an idea, let $X=T^{2}$ be the two-dimensional torus. One can regard $T^{2}$ as the quotient of the Euclidean plane $\mathbb{R}^{2}$ by the integer lattice $\mathbb{Z}^{2}$, endowed with a fixed orientation. Since $\pi_{1}\left(T^{2}\right)=\mathbb{Z} \oplus \mathbb{Z}$, the automorphisms of $T^{2}$ correspond to the elements of group $G L_{2}(\mathbb{Z})$ as any element $A \in G L_{2}(\mathbb{Z})$ maps $\mathbb{Z}^{2}$ to itself and so induces a continuous map $\varphi_{A}: T^{2} \rightarrow T^{2}$; the map $\varphi_{A}$ has an inverse $\varphi_{A}^{-1}$ given by the matrix $A^{-1}$. The map $\varphi_{A}$ preserves orientation if and only if $\operatorname{det}(A)=1$; thus $\operatorname{Mod}\left(T^{2}\right) \cong S L_{2}(\mathbb{Z})$. If $A \in S L_{2}(\mathbb{Z})$, the characteristic polynomial of $A$ can be written as $\lambda^{2}-(\operatorname{tr}(A)) \lambda+1$. The eigenvalues are either: (i) complex, i.e. $\operatorname{tr}(A)=0$ or \pm 1 ; (ii) double reals +1 or -1 , i.e. $\operatorname{tr}(A)=2$ or -2 ; (iii) distinct reals, i.e. $|\operatorname{tr}(A)|>2$. At the level of surface automorphisms, the above cases correspond to: (i) a finite order automorphism $\left(\varphi_{A}\right)^{12}=1$; (ii) matrix $A$ has an integral eigenvector which defines a measured foliation $\mathcal{F}$ on $T^{2}$. The leaves of $\mathcal{F}$ are closed and invariant under the map $\varphi_{A}$; the latter is a Dehn twist in $C \in \mathcal{F}$. 
The automorphism $\varphi_{A}$ is parabolic and $\mathcal{F}$ is called a Strebel foliation. (iii) $\lambda_{A}>1>1 / \lambda_{A}$, where $\lambda_{A}$ is the Perron-Frobenius eigenvalue and we let $v_{A}, v_{A}^{\prime}$ be the corresponding eigenvectors. Since $\theta_{A}=v_{A}^{(2)} / v_{A}^{(1)}$ is irrational, $\varphi_{A}$ leaves no simple closed curve invariant. The translation of vectors $v_{A}$ and $v_{A}^{\prime}$ yields two measured foliations $\mathcal{F}_{u}$ and $\mathcal{F}_{s}$, which are carried by $\varphi_{A}$ to the foliations $\lambda_{A} \mathcal{F}_{u}$ and $\frac{1}{\lambda_{A}} \mathcal{F}_{s}$, respectively. In other words, $\varphi_{A}$ is a linear homeomorphism which stretches by a factor $\lambda_{A}$ in one direction and shrinks by the same factor in a complementary direction. The homeomorphism $\varphi_{A}$ is hyperbolic and invariant foliations $\mathcal{F}_{s}$ and $\mathcal{F}_{u}$ are called Anosov foliations. What is remarkable, the classification holds for the higher genus surfaces; due to natural restrictions we can only outline these results referring the interested reader to the excellent monograph [Casson \& Bleilier 1988] [2]. It is proved that each automorphism $\varphi \in \operatorname{Mod}(X)$ is isotopic to an automorphism $\varphi^{\prime}$, such that either: (i) $\varphi^{\prime}$ has finite order; (ii) $\varphi^{\prime}$ is an infinite order parabolic automorphism and preserves a Strebel foliation; (iii) $\varphi^{\prime}$ is of infinite order and does not preserve the simple closed curves on $X$. In this case there exist a stable $\mathcal{F}_{s}$ and unstable $\mathcal{F}_{u}$ mutually orthogonal measured foliations on $X$ such that $\varphi^{\prime}\left(\mathcal{F}_{s}\right)=\frac{1}{\lambda_{\varphi^{\prime}}} \mathcal{F}_{s}$ and $\varphi^{\prime}\left(\mathcal{F}_{u}\right)=\lambda_{\varphi^{\prime}} \mathcal{F}_{u}$, where $\lambda_{\varphi^{\prime}}>1$ is called the dilatation of $\varphi^{\prime}$. The foliations $\mathcal{F}_{s}, \mathcal{F}_{u}$ are minimal, uniquely ergodic and describe $\varphi^{\prime}$ up to a power. The automorphism $\varphi^{\prime}$ is hyperbolic and invariant foliations $\mathcal{F}_{s}, \mathcal{F}_{u}$ are called pseudo-Anosov foliations.

\section{Proofs}

\subsection{Proof of lemma 1}

(i) For brevity, let $\mathfrak{m}:=\operatorname{Jac}(\mathcal{F})$; suppose that $A=\left(a_{i j}\right) \in G L_{n}(\mathbb{Z})$ and $\gamma_{i}^{\prime}=\sum_{j=1}^{n} a_{i j} \gamma_{j}$ is a new basis in $H_{1}(X, \operatorname{Sing} \mathcal{F} ; \mathbb{Z})$. We have to show, that $\mathfrak{m}$ does not depend on the new basis; indeed, using the integration rules we get:

$$
\lambda_{i}^{\prime}=\int_{\gamma_{i}^{\prime}} \phi=\int_{\sum_{j=1}^{n} a_{i j} \gamma_{j}} \phi=\sum_{j=1}^{n} \int_{\gamma_{j}} \phi=\sum_{j=1}^{n} a_{i j} \lambda_{j} .
$$

To prove that $\mathfrak{m}=\mathfrak{m}^{\prime}$, consider the following equations:

$$
\mathfrak{m}^{\prime}=\sum_{i=1}^{n} \mathbb{Z} \lambda_{i}^{\prime}=\sum_{i=1}^{n} \mathbb{Z} \sum_{j=1}^{n} a_{i j} \lambda_{j}=\sum_{j=1}^{n}\left(\sum_{i=1}^{n} a_{i j} \mathbb{Z}\right) \lambda_{j} \subseteq \mathfrak{m} .
$$


Let $A^{-1}=\left(b_{i j}\right) \in G L_{n}(\mathbb{Z})$ be an inverse to the matrix $A$. Then $\lambda_{i}=$ $\sum_{j=1}^{n} b_{i j} \lambda_{j}^{\prime}$ and

$$
\mathfrak{m}=\sum_{i=1}^{n} \mathbb{Z} \lambda_{i}=\sum_{i=1}^{n} \mathbb{Z} \sum_{j=1}^{n} b_{i j} \lambda_{j}^{\prime}=\sum_{j=1}^{n}\left(\sum_{i=1}^{n} b_{i j} \mathbb{Z}\right) \lambda_{j}^{\prime} \subseteq \mathfrak{m}^{\prime} .
$$

Since both $\mathfrak{m}^{\prime} \subseteq \mathfrak{m}$ and $\mathfrak{m} \subseteq \mathfrak{m}^{\prime}$, we conclude that $\mathfrak{m}^{\prime}=\mathfrak{m}$. Part (i) of lemma 1 follows.

(ii) Let $h: X \rightarrow X$ be an automorphism of surface $X$. Denote by $h_{*}$ its action on $H_{1}(X, \operatorname{Sing}(\mathcal{F}) ; \mathbb{Z})$ and by $h^{*}$ on $H^{1}(X ; \mathbb{R})$ connected by the formula:

$$
\int_{h_{*}(\gamma)} \phi=\int_{\gamma} h^{*}(\phi), \quad \forall \gamma \in H_{1}(X, \operatorname{Sing}(\mathcal{F}) ; \mathbb{Z}), \quad \forall \phi \in H^{1}(X ; \mathbb{R}) .
$$

Let $\phi, \phi^{\prime} \in H^{1}(X ; \mathbb{R})$ be the closed forms, whose trajectories define the foliations $\mathcal{F}$ and $\mathcal{F}^{\prime}$, respectively. Since $\mathcal{F}, \mathcal{F}^{\prime}$ are topologically conjugate, $\phi^{\prime}=\mu h^{*}(\phi)$ for a $\mu>0$. Let $\operatorname{Jac}(\mathcal{F})=\mathbb{Z} \lambda_{1}+\ldots+\mathbb{Z} \lambda_{n}$ and $\operatorname{Jac}\left(\mathcal{F}^{\prime}\right)=$ $\mathbb{Z} \lambda_{1}^{\prime}+\ldots+\mathbb{Z} \lambda_{n}^{\prime}$. Then:

$$
\lambda_{i}^{\prime}=\int_{\gamma_{i}} \phi^{\prime}=\mu \int_{\gamma_{i}} h^{*}(\phi)=\mu \int_{h_{*}\left(\gamma_{i}\right)} \phi, \quad 1 \leq i \leq n .
$$

By item (i), it holds:

$$
\operatorname{Jac}(\mathcal{F})=\sum_{i=1}^{n} \mathbb{Z} \int_{\gamma_{i}} \phi=\sum_{i=1}^{n} \mathbb{Z} \int_{h_{*}\left(\gamma_{i}\right)} \phi .
$$

Therefore:

$$
\operatorname{Jac}\left(\mathcal{F}^{\prime}\right)=\sum_{i=1}^{n} \mathbb{Z} \int_{\gamma_{i}} \phi^{\prime}=\mu \sum_{i=1}^{n} \mathbb{Z} \int_{h_{*}\left(\gamma_{i}\right)} \phi=\mu \operatorname{Jac}(\mathcal{F}) .
$$

Lemma 1 follows.

\subsection{Proof of theorem 1}

We shall split the proof in a series of lemmas, starting with the following elementary

Lemma 2 Let $T \in M_{n}(\mathbb{Z})$ be an endomorphism of the vector space $\mathbb{R}^{n}$, where

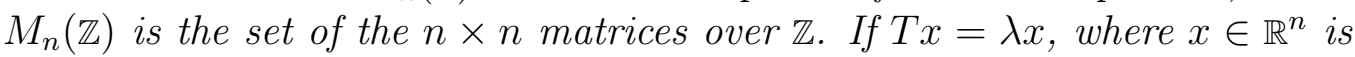
an eigenvector and $\lambda \in K$ is an eigenvalue of $T$, then $x$ can be scaled so that $x \in K^{n}$, where $K=\mathbb{Q}(\lambda)$ is a subfield of $\mathbb{R}$ generated by $\lambda$. 
Proof. The proof is left to the reader as an exercise in linear algebra; otherwise, we refer to the results of [Borevich \& Shafarevich 1966, Chapter 2, Section 2] [1].

Let $f_{N} \in S_{2}\left(\Gamma_{0}(N)\right)$ be a (normalized) Hecke eigenform, such that $f_{N}(z)=$ $\sum_{n=1}^{\infty} c_{n}\left(f_{N}\right) q^{n}$ its Fourier series. We shall denote by $K_{f}=\mathbb{Q}\left(\left\{c_{n}\left(f_{N}\right)\right\}\right)$ the algebraic number field generated by the Fourier coefficients of $f_{N}$. If $g$ is the genus of the modular curve $X_{0}(N)$, then $1 \leq \operatorname{deg}\left(K_{f} \mid \mathbb{Q}\right) \leq g$ and $K_{f}$ is a totally real field [Darmon 2004] [3], p. 25. Let $\mathcal{F}_{N}$ be the foliation by vertical trajectories of $f_{N}$. The following lemma will be critical.

Lemma $3 \operatorname{Jac}\left(\mathcal{F}_{N}\right)$ is a $\mathbb{Z}$-module in the field $K_{f}$.

Proof. Let $\phi_{N}=\Re\left(f_{N} d z\right)$, where $f_{N}$ is the Hecke eigenform. By the definition, Jac $\left(\mathcal{F}_{N}\right)=\int_{H_{1}\left(X_{0}(N), \operatorname{Sing} \mathcal{F}_{N} ; \mathbb{Z}\right)} \phi_{N}=\mathbb{Z} \lambda_{1}+\ldots+\mathbb{Z} \lambda_{g}, \lambda_{i} \in \mathbb{R}$. Let us study the action of the Hecke operators on the $\operatorname{Jac}\left(\mathcal{F}_{N}\right)$. Since $f_{N}$ is an eigenform, $T_{n} f_{N}=c_{n} f_{N}, \forall T_{n} \in \mathbb{T}_{\mathbb{Z}}$. By virtue of the isomorphism $S_{2}\left(\Gamma_{0}(N)\right) \cong$ $\Omega_{h o l}\left(X_{0}(N)\right)$, one gets $T_{n} \omega_{N}=c_{n} \omega_{N}$, where $c_{n} \in K_{f}$. Let us evaluate the real parts of the last equation as follows: $\Re\left(T_{n} \omega_{N}\right)=T_{n}\left(\Re \omega_{N}\right)=\Re\left(c_{n} \omega_{N}\right)=$ $c_{n}\left(\Re \omega_{N}\right)$; note that the equality $\Re\left(c_{n} \omega_{N}\right)=c_{n}\left(\Re \omega_{N}\right)$ involves the fact that $c_{n}$ is a real number. One concludes that $T_{n} \phi_{N}=c_{n} \phi_{N}, \forall T_{n} \in \mathbb{T}_{\mathbb{Z}}$ and $c_{n} \in K_{f}$; therefore, the action of the Hecke operator $T_{n}$ on the $\operatorname{Jac}\left(\mathcal{F}_{N}\right)$ can be written as:

$$
T_{n}\left(J a c\left(\mathcal{F}_{N}\right)\right)=\int_{H_{1}} T_{n} \phi_{N}=\int_{H_{1}} c_{n} \phi_{N}=c_{n} J a c\left(\mathcal{F}_{N}\right), \quad c_{n} \in K_{f},
$$

where $H_{1}=H_{1}\left(X_{0}(N)\right.$, Sing $\left.\mathcal{F} ; \mathbb{Z}\right)$ is the relative homology group. Thus, $T_{n}$ multiplies $\operatorname{Jac}\left(\mathcal{F}_{N}\right)$ by the real number $c_{n}$.

Consider the $\mathbb{Z}$-module $\operatorname{Jac}\left(\mathcal{F}_{N}\right)=\mathbb{Z} \lambda_{1}+\ldots+\mathbb{Z} \lambda_{g}$, where $\lambda_{i} \in \mathbb{R}$. The Hecke operator $T_{n}$ is an endomorphism of the vector space $\left\{\lambda \in \mathbb{R}^{g} \mid \lambda=\right.$ $\left.\left(\lambda_{1}, \ldots, \lambda_{g}\right)\right\}$. By virtue of (10), vector $\lambda \in \mathbb{R}^{g}$ is an eigenvector of the linear operator $T_{n} \in M_{g}(\mathbb{Z})$. In other words, $T_{n} \lambda=c_{n} \lambda, c_{n} \in K_{f}$. In view of lemma 2, one can scale the vector $\lambda$ so that $\lambda_{i} \in K_{f}$.

$\operatorname{Lemma} 4 \operatorname{rank}\left(\operatorname{Jac}\left(\mathcal{F}_{N}\right)\right)=\operatorname{deg}\left(K_{f} \mid \mathbb{Q}\right)$.

Proof. Let $I_{f}=\left\{T \in \mathbb{T}_{\mathbb{Z}}: T f=0\right\}$ and $R=\mathbb{T}_{\mathbb{Z}} / I_{f}$. It is well known that $R$ is an order in the number field $K_{f}$, i.e. a subring of the ring of integers of $K_{f}$ containing 1 . Recall that a coefficient ring of the lattice $\Lambda$ in $K_{f}$ is defined as 
the set $\alpha \in K_{f}$, such that $\alpha \Lambda \subseteq \Lambda$. The coefficient ring is always an order in $K_{f}$. It is verified directly, that $R$ is the coefficient ring of $\operatorname{Jac}\left(\mathcal{F}_{N}\right)$. Moreover, $\operatorname{Jac}\left(\mathcal{F}_{N}\right)$ can be scaled so that $\operatorname{Jac}\left(\mathcal{F}_{N}\right) \subseteq R$ [Borevich \& Shafarevich 1966] [1], p.88. Therefore, Jac $\left(\mathcal{F}_{N}\right)$ and $R$ have the same number of generators, i.e. the same rank ibid. But rank $(R)=\operatorname{deg}\left(K_{f} \mid \mathbb{Q}\right)[$ Diamond \& Shurman 2005] [4, p.234. Thus, $\operatorname{rank}\left(\operatorname{Jac}\left(\mathcal{F}_{N}\right)\right)=\operatorname{deg}\left(K_{f} \mid \mathbb{Q}\right)$.

Lemma 5 The foliation $\mathcal{F}_{N}$ is topologically conjugate to:

(i) a Strebel foliation, if deg $\left(K_{f} \mid \mathbb{Q}\right)=1$;

(ii) a pseudo-Anosov foliation, if deg $\left(K_{f} \mid \mathbb{Q}\right)=g$;

(iii) a degenerate pseudo-Anosov foliation, if $2 \leq \operatorname{deg}\left(K_{f} \mid \mathbb{Q}\right) \leq g-1$.

Proof. We shall use the well known construction of measured foliations from the generators $\lambda_{i}>0$ of their Jacobians; the construction is known as a zippering of the rectangles [Veech 1982] [8] and runs (roughly) as follows. Let $\lambda_{1}, \ldots, \lambda_{n}$ be positive generators of $\operatorname{Jac}(\mathcal{F})$ and fix a permutation $\pi$ of $n$ symbols; let $R_{i}$ be $n$ rectangles with the base $\lambda_{i}$ placed one-next-to-other in $\mathbb{R}^{2}$ and foliated by the vertical lines. One glues the bottom $\lambda_{i}$ of $R_{i}$ with the top $\lambda_{\pi(i)}$ of $R_{\pi(i)}$; since the permutation $\pi$ preserves the total length $\lambda_{1}+\ldots+\lambda_{n}$ of the intervals, all rectangles will be glued in this way. By zippering of the remaining vertical sides of $R_{i}$ one gets a compact surface $X$ with the measured foliation $\mathcal{F}$ on it; by the construction $J a c(\mathcal{F})$ is generated by $\lambda_{i}$.

In fact, the Veech construction gives a little more than just a foliation on compact surface; the surface comes with a complex structure, see [Veech 1982] [8], p. 215. Our argument does not use the complex structure but the reader can verify (given the arithmetic nature of periods $\lambda_{i}$ ) that the underlying Riemann surface is arithmetic, i.e. the orbit space of an arithmetic Fuchsian group.

(i) Let $\operatorname{deg}\left(K_{f} \mid \mathbb{Q}\right)=1$, i.e the minimal possible. By lemma 3 , $\operatorname{rank}\left(\operatorname{Jac}\left(\mathcal{F}_{N}\right)\right)=1$ and therefore scaled $\lambda_{i}$ are all rational. Since $\lambda_{i}$ are linearly dependent over $\mathbb{Q}$, the zippered rectangles will produce a measured foliation all of whose leaves, but a finite number, are closed. Such a foliation is traditionally called the Strebel foliation [Strebel 1984] [6].

(ii) Let $\operatorname{deg}\left(K_{f} \mid \mathbb{Q}\right)=g$ be the maximal possible; in this case $\lambda_{i}$ are linearly independent over $\mathbb{Q}$. Thus, $\mathcal{F}_{N}$ is a minimal foliation, i.e. each non-singular leaf of $\mathcal{F}_{N}$ is dense on the surface $X_{0}(N)$. To show that $\mathcal{F}$ 
is a pseudo-Anosov foliation, let $\lambda_{i}>0$ and $A \in G L_{g}(\mathbb{Z})$ be a positive matrix with the Perron-Frobenius eigenvector $\left(\lambda_{1}, \ldots, \lambda_{g}\right)$. Denote by $i$ an involution, which acts on $S_{2}\left(\Gamma_{0}(N)\right)$ according with the formula

$$
f(z)=\sum c_{n} q^{n} \longmapsto f^{*}(z)=\sum \bar{c}_{n} q^{n}
$$

where the bar sign means complex conjugation; since $S_{2}\left(\Gamma_{0}(N)\right) \cong H^{1}\left(X_{0}(N) ; \mathbb{R}\right)$, the involution defines a split $H^{1}\left(X_{0}(N) ; \mathbb{R}\right)=$ $H_{\mathbb{R}}^{1} \oplus H_{i \mathbb{R}}^{1}$. The matrix $A$ acts on $H_{\mathbb{R}}^{1}$ and extends to the whole space $H^{1}\left(X_{0}(N) ; \mathbb{R}\right)$ by the involution $i$; the corresponding Perron-Frobenius eigenvector has the form $\left(\lambda_{1}, \ldots, \lambda_{g}, \lambda_{1}, \ldots, \lambda_{g}\right)$. By the argument of [Thurston 1988] [7] on p.427, one concludes that there exists a pseudo-Anosov automorphism $\varphi_{A}: X_{0}(N) \rightarrow X_{0}(N)$, such that $\varphi_{A}^{*}=A$; in other words, $\mathcal{F}_{N}$ is an invariant foliation of the automorphism $\varphi_{A}$.

(iii) Let $\operatorname{deg}\left(K_{f} \mid \mathbb{Q}\right)=n$, where $2 \leq n \leq g-1$; in this case there are only $n$ rationally independent $\lambda_{i}$ among $\lambda_{1}, \ldots, \lambda_{g}$. The foliation $\mathcal{F}_{N}$ is still minimal, since $n>1$; however, it will acquire $g-n$ extra separatrix connections, i.e leaves joining the saddle points of $\mathcal{F}_{N}$. We repeat the argument of item (ii) to prove that $\mathcal{F}_{N}$ is a pseudo-Anosov foliation, but because of presence of the separatrix connections it will be a degenerate one.

Theorem 1 follows from lemma 5 .

Acknowledgments. I am grateful to Lawrence D. Taylor (University of Nottingham) for useful correspondence and to a referee for helpful comments.

\section{References}

[1] Z. I. Borevich and I. R. Shafarevich, Number Theory, Acad. Press, 1966.

[2] A. J. Casson and S. A. Bleiler, Automorphisms of Surfaces after Nielsen and Thurston, Lond. Math. Soc. Student Texts 9, Cambridge, 1988.

[3] H. Darmon, Rational Points on Modular Elliptic Curves, CBMS Regional Conference Series 101, Amer. Math. Soc., Providence, RI, 2004.

[4] F. Diamond and J. Shurman, A First Course in Modular Forms, GTM 228, Springer, 2005 
[5] H. B. Lawson, Foliations, Bull. Amer. Math. Soc. 80 (1974), 369-418.

[6] K. Strebel, Quadratic Differentials, Springer, 1984.

[7] W. P. Thurston, On the geometry and dynamics of diffeomorphisms of surfaces, Bull. Amer. Math. Soc. 19 (1988), 417-431.

[8] W. A. Veech, Gauss measures for transformations on the space of interval exchange maps, Ann. of Math. (2) 115 (1982), no. 1, 201-242.

The Fields Institute for Research in Mathematical Sciences, Toronto, ON, CANAdA, E-MAIL: igor.v.nikolaev@gmail.com

Current address: Department of Mathematics, University of Sherbrooke, Igor.Nikolaev@USherbrooke.ca 